\title{
Zwischen Kriminalisierung und Regulierung: Der schwindende Aktionsradius der zivilen Seenotrettungsorganisationen im Mittelmeer - ein Eingriff in die Vereinigungsfreiheit?
}

\begin{abstract}
Salome Schmid *
Die Situation der zivilgesellschaftlichen Such- und Rettungsorganisationen im Mittelmeer spitzt sich zu: Vorwürfe zur Zusammenarbeit mit Schleppern, Verweigerung des Hafenzugangs, Beschlagnahmungen von Rettungsbooten und Ermittlungen gegen Mitarbeitende sind keine Seltenheit - dies trotz ihrer wichtigen Rolle in der Rettung von Menschenleben und einer bestehenden völker- und menschenrechtlichen Pflicht zur Seenotrettung. Im Zusammenspiel mit Regulierungsversuchen wie dem italienischen Verhaltenskodex und weiteren Massnahmen führt dies dazu, dass der Aktionsradius zivilgesellschaftlicher Such- und Rettungsorganisationen auf der zentralen Mittelmeerroute schwindet. Die EU scheint bereit zu sein, im Rahmen des von der EU Kommission formulierten Ziels der «Verringerung der Migrationsströme» selbst den Schutz fundamentaler Menschenrechte bzw. die Rettung von Menschenleben in den Hintergrund treten zu lassen. Den zivilgesellschaftlichen Such- und Rettungsorganisationen wird es nahezu verunmöglicht, ihre Einsätze unter Wahrung der humanitären Prinzipien durchzuführen. Der folgende Beitrag zeigt auf, dass dies eine Verletzung der Vereinigungsfreiheit darstellt, auf deren Schutz sich die zivilen Seenotrettungsorganisationen im Mittelmeer berufen können.
\end{abstract}

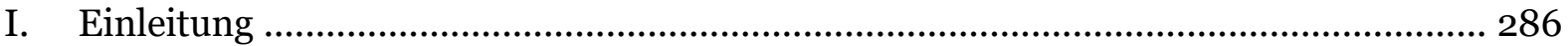

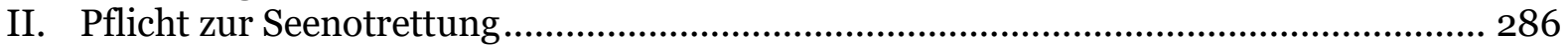

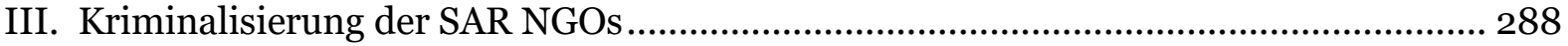

1. Tatbestand der Schlepperei im Völkerrecht .................................................... 288

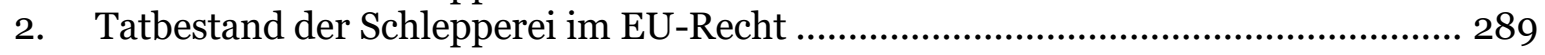

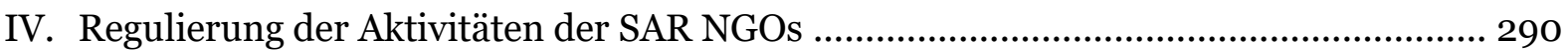

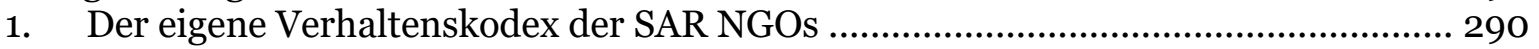

2. Der italienische Verhaltenskodex für SAR NGOs ........................................................ 290

V. Zur Vereinigungsfreiheit von SAR NGOs im Mittelmeer .............................................292

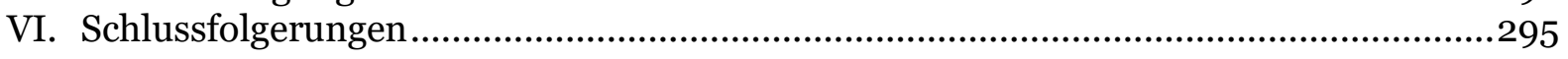

Zitiervorschlag: Salome Schmid, Zwischen Kriminalisierung und Regulierung: Die Situation der zivilen Seenotrettungsorganisationen im Mittelmeer - ein Eingriff in die Vereinigungsfreiheit?, in: sui-generis 2018, S. 285

URL: $\quad$ sui-generis.ch/74

DOI: $\quad$ https://doi.org/10.21257/sg.74

* Salome Schmid (salome.schmid[at]hotmail.com), MLaw, verfasst als Assistentin am Lehrstuhl für Europarecht und europäisches Migrationsrecht der Universität Fribourg (Prof. S. ProginTheuerkauf). Diese Publikation entstand im Rahmen des Nationalen Forschungsschwerpunktes «nccr - on the move» finanziert vom Schweizerischen Nationalfonds und basiert auf Recherchen, welche die Autorin im Rahmen eines zweimonatigen Forschungsaufenthaltes an der Queen Mary University of London (Jan./Feb. 2018) absolvierte. Ein besonderer Dank geht dabei an Sarah Progin-Theuerkauf, Constantin Hruschka und Violeta Moreno-Lax für die wertvollen Anmerkungen und die Hilfestellungen beim Verfassen des Beitrages.

Dieses Werk ist lizenziert unter einer Creative Commons Namensnennung - Weitergabe unter gleichen Bedingungen 4.0 International Lizenz. 


\section{Einleitung}

1 Auch im Jahr 2017 forderte die zentrale Mittelmeerroute als Fluchtweg nach Europa zahlreiche Todesopfer. ${ }^{1}$ Trotz oder zum Teil auch gerade wegen ihrer wichtigen Rolle bei der Seenotrettung standen die im Mittelmeer tätigen zivilgesellschaftlichen Organisationen ${ }^{2}$ immer wieder stark in der Kritik. Ihre Tätigkeiten wurden öffentlich mit den Aktivitäten von Schleppern in Verbindung gebracht und es wurde gegen in der zivilen Seenotrettung involvierte Personen rechtlich vorgegangen. ${ }^{3}$ Diese Entwicklung zeigt sich auch in den laufenden Ermittlungen gegen Mitarbeitende der spanischen Nichtregierungsorganisation (NGO) «Proactiva» und der erfolgten Beschlagnahmung ihres Rettungsbootes «Open Arms».4 Als Reaktion auf diese Ereignisse forderte die Europäische Kommission die Parteien lediglich erneut zur Einhaltung des umstrittenen italienischen Verhaltenskodex für die in der Seenotrettung tätigen NGOs auf.5

2 Der folgende Beitrag beschäftigt sich mit der rechtlichen wie auch der faktischen Situation von zivilgesellschaftlichen Such- und Rettungsorganisationen (SAR ${ }^{6}$

1 IOM, Pressemitteilung vom 1 Mai 2018, 2832 Todesopfer auf der zentralen Mittelmeerroute im Jahr 2017.

2 Im Folgenden wird auch der Begriff der NGO (Non-Governmental Organisation) verwendet.

3 Siehe unter II. Kriminalisierung der SAR NGOs.

4 Das Rettungsboot «Open Arms» wurde nach mehreren Wochen wieder freigegeben, die Ermittlungen gegen die Mitarbeitenden von «Proactiva» sind jedoch weiterhin am Laufen; vgl. ECRE, Proactiva rescue ship released, crew members remain under investigation, 20. April 2018; vgl. zum ganzen Fall: Statewatch, Analysis, The Seizure of the Open Arm Boat as a Paradigm of the war against human rights, April 2018.

5 ANSA, Respect Migrant-Rescue Conduct CodeEU, 19. März 2018; vgl. Statewatch (Fn. 4), S. 9 f.

6 Aus dem Englischen Search And Rescue (SAR).
NGOs) im Mittelmeer. Nach einer Einleitung in die Thematik der Seenotrettung beleuchtet der Beitrag die Phänomene der Kriminalisierung und Regulierung ihrer Aktivitäten, um deren Zusammenspiel aufzuzeigen. Anschliessend wird die Vereinbarkeit dieser Entwicklung mit der Vereinigungsfreiheit der SAR NGOs am Beispiel des italienischen Verhaltenskodex' geprüft.

\section{Pflicht zur Seenotrettung}

3 Die Pflicht zur Seenotrettung ist ein Grundprinzip des internationalen Seerechts und findet sich in verschiedenen Übereinkommen, namentlich dem UN-Seerechtsübereinkommen (SRÜ)7, dem internationalen Übereinkommen zum Schutze des menschlichen Lebens auf See $(S O L A S)^{8}$ und dem internationalen Übereinkommen über Seenotrettung (SARÜbereinkommen)9 .10 Art. 98 SRÜ verpflichtet einerseits die Küstenstaaten, effektive Such- und Rettungsdienste zu errichten und so für «die Sicherheit auf und über der See» zu sorgen; andererseits müssen die Flaggenstaaten die Kapitäne ihrer Schiffe in die Pflicht nehmen, Per-

7 Seerechtsübereinkommen der Vereinten Nationen vom 10. Dezember 1982 (SR 0.747.305.15).

8 Internationales Übereinkommen von 1974 zum Schutze des menschlichen Lebens auf See vom 1. November 1974 (SR 0.747.363.33).

9 Internationales Übereinkommen über Seenotrettung vom 27. April 1979 (no. 23489).

10 Violeta Moreno-Lax, Seeking Asylum in the Mediterranean: Against a Fragmentary Reading of EU Obligations Accruing at Sea, in: International Journal of Refugee Law, 23, 2011, S. 194 (zit.: Moreno-Lax, Seeking Asylum); Sarah ProginTheuerkauf, Rechtsrahmen der Seenotrettung in der EU, in: Zeitschrift für Europarecht, 2015, S. 72 und 77; Irini Papanicolopulu, the duty to rescue at sea, in peacetime and in war: a general overview, in: International Review of the Red Cross, 2016, S. 492 und 494. 
sonen in Seenot Hilfe zu leisten. ${ }^{11}$ Diese völkerrechtliche Rettungspflicht gilt unterschiedslos gegenüber allen Menschen in Seenot. ${ }^{12}$ Die geretteten Personen müssen nach erfolgter Rettung an einen «sicheren Ort» 13 gebracht werden. ${ }^{14}$ Jedoch fehlen eindeutige Regelungen über diesen «sicheren Ort» bzw. über die Bestimmung des Staates, der für die Aufnahme der geretteten Personen zuständig ist. ${ }^{15}$ Aus dieser fehlenden klaren rechtlichen Regelung entstehen Unsicherheiten in der Rechtsanwendung, die von den Staaten teilweise als Handlungsspielraum ausgenutzt werden. ${ }^{16}$ Wie die aktuellen Beispiele der «Aquarius» und «Lifeline» zeigen, birgt dies die Gefahr, dass dem Rettungsboot samt geretteten Personen das Hafenzugangsrecht verweigert wird. ${ }^{17}$ Dieser faktische Handlungsspielraum der Staaten ist insbesondere deswegen problematisch, da bei Personen, von denen vermutet wird, dass sie internationalen Schutz beantragen wollen, die Aufnahmebereitschaft der Staaten geringer zu sein scheint und da-

11 Papanicolopulu (Fn. 10), S. 493.

12 Vgl. Art. 98 Abs. 1 lit. a SRÜ: «jeder Person [...] [ist] Hilfe zu leisten», Moreno-Lax, Seeking Asylum (Fn. 10), S. 195; Wissenschaftliche Dienste Deutscher Bundestag, Völkerrechtliche Schutzpflichten gegenüber Migranten in Seenot, 31. Oktober 2013, S. 5 (zit.: Wissenschaftliche Dienste Deutscher Bundestag, Seenot).

13 SAR, Anlage, Kapitel 1.3.2.

14 Sicco Rah, Asylsuchende und Migranten auf See, Staatliche Rechte und Pflichten aus völkerrechtlicher Sicht, 2009, S. 110; Papanicolopulu (Fn. 10), S. 499 f.

15 Vgl. Moreno-Lax, Seeking Asylum (Fn. 10), S. 196 ff.; Rah (Fn. 14), S. 103 f. und 110; Papanicolopulu (Fn. 10), S. 501.

16 Vgl. Moreno-Lax, Seeking Asylum (Fn. 10), S. 198 ff.; Papanicolopulu (Fn. 10), S. 499 ff.

17 Vgl.: NZZ, Nach der «Aquarius» nun die «Lifeline»: Flüchtlingsschiff sitzt auf dem Mittelmeer fest, 23. Juni 2018; vgl. Cap Anamur beschrieben in: Tugba Basaran, The saved and the drowned: Governing indifference in the name of security, Security Dialogue, 46(3), 2015, S. 211; Papanicolopulu (Fn. 10), S. 500; Rah (Fn. 14), S. 104 ff. mit auch Probleme mit dem Zugang zu einem Asylverfahren verbunden sind. ${ }^{18}$ Zudem droht dem Kapitän und ggf. der Crew bei Missachtung der Verweigerung des Hafenzuganges eine Strafverfolgung. ${ }^{19}$

4 Die Pflicht zur Seenotrettung ergibt sich auch aus menschenrechtlichen Garantien.20 Im europäischen Kontext kommt dabei insbesondere der EMRK ${ }^{21}$ erhebliche Bedeutung zu. ${ }^{22}$ Das Recht auf Leben (insb. verankert in Art. 2 EMRK und Art. 2 GRC23) verpflichtet die Mitgliedstaaten sowie die Organe und Institutionen der EU24, Menschen in Seenot Hilfeleistungen zu gewähren, sowie durch Such- und Rettungsoperationen das menschliche Leben im Mittelmeerraum aktiv zu schützen. ${ }^{25}$ Das NonRefoulement-Prinzip (Art. 3 EMRK,

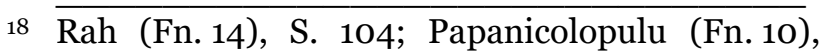
S. 500; siehe auch: Lisa-Marie Komp, the Duty to Assist Persons in Distress: An Alternative Source of Protection against the Return of Migrants and Asylum Seekers to the High Seas?, in: Boat Refugees' and Migrants at Sea: A Comprehensive Approach, Integrating Maritime Security with $\mathrm{Hu}-$ man Rights, 2017, S. 231.

19 Mehr dazu im nachfolgenden Kapitel III. Kriminalisierung der SAR NGOs; vgl. Cap Anamur, Basaran (Fn. 17), S. 211 f.

20 Rah (Fn. 14), S. 210 ff.; Wissenschaftliche Dienste Deutscher Bundestag, Seenot (Fn. 12), S. 7 ff.; vgl. Progin-Theuerkauf (Fn. 10), S. $71 \mathrm{f}$.

21 Konvention zum Schutze der Grundfreiheiten und Menschenrechte vom 4. November 1950 (SR 0.101).

22 Rah (Fn. 14), S. 211.

23 Charta der Grundrechte der Europäischen Union vom 18. Dezember 2010 (200/C 364/1).

24 Alle EU-Mitgliedstaaten sind Vertragsstaaten der EMRK, die EU selbst ist der EMRK nicht beigetreten. Die Grundrechtscharta als Teil des Primärrechts, gilt jedoch sowohl für die Organe und Institutionen der EU, wie auch die Mitgliedstaaten bei der Anwendung von EU-Recht (vgl. Art. 6 EUV); vgl. auch Art. 51 i.V.m. Art. 52 Abs. 3 GRC; Progin-Theuerkauf (Fn. 10), S. 73.

25 Progin-Theuerkauf (Fn. 10), S. 72 f.; Papanicolopulu (Fn. 10), S. 511; Rah (Fn. 14), S. 218 f.; Siehe ausführlich: Komp (Fn. 18), S. 236 ff. 
Art. 33 GFK $^{26}$ ) verbietet ausserdem, die betroffenen Menschen auf dem Seeweg in Länder zurückzuweisen, in welchen ihnen Folter oder unmenschliche oder erniedrigende Strafe oder Behandlung drohen. ${ }^{27}$

\section{Kriminalisierung der SAR NGOs}

5 Trotz des Bestehens der völker- und menschenrechtlichen Seenotrettungspflicht wurden die Tätigkeiten von NGOs im Seenotrettungsbereich vermehrt kritisiert und mit Vorwürfen zur Zusammenarbeit mit Schleppern konfrontiert. ${ }^{28}$ In jüngster Zeit wurden in zwei bekannten Fällen Rettungsschiffe von SAR NGOs beschlagnahmt. ${ }^{29}$ In Griechenland stand zudem die Verhandlung gegen Mitarbeitende einer spanischen NGO an, welche des Menschenschmuggels beschuldigt,

26 Abkommen über die Rechtsstellung der Flüchtlinge vom 28. Juli 1951 (Genfer Flüchtlingskonvention; SR 0.142.30).

27 Progin-Theuerkauf (Fn. 10), S. 71; Komp (Fn. 18), S. 225; Rah (Fn. 14), S. 213 ff.; vgl. auch: Urteil des EGMR 27765/o9 vom 23. Februar 2012 (Hirsi Jamaa gegen Italien); Violeta Moreno-Lax, Hirsi Jamaa and Others v Italy or Strasbourg Court versus Extraterritorial Migration Control?, Human Rights Law Review, 12, 2012, S. 574-598.

28 Vgl. Daniel Ghezelbash/Violeta Moreno-Lax/ Natalie Klein/Brian Opeskin, Securitization of Search and Rescue at Sea: The Response to Boat Migration in the Mediterranean and Offshore Australia, British Institute of International and Comparative Law, 67(4), 2018, S. $347 \mathrm{ff}$.

29 Die spanische NGO Proactiva, sowie die deutsche NGO «Jugend rettet»; Pressemitteilung Jugend rettet, Parallelen zwischen Beschlagnahmen der Rettungsschiffe Open Arms und IUVENTA, 21. März 2018; Ghezelbash/Moreno-Lax/Klein/ Opeskin (Fn. 28), S. 347; Eugenio Cusumano, The sea as humanitarian space. Nongovernmental Search and Rescue dilemmas on the Central Mediterranean migratory route, Mediterranean Politics vom 16. März 2017, S. 2 f. (zit.: Cusumano, humanitarian sea); vgl. Eugenio Cusumano, Straightjacketing migrant rescuers? The Code of Conduct on maritime NGOs, Mediterranean Politics vom 17. September 2017, S. 2 f. (zit.: Cusumano, Straightjacketing). schliesslich jedoch freigesprochen wurden. $3^{\circ}$ Diese Ereignisse zeigen die (reale) Gefahr der Kriminalisierung der Seenotrettungsaktivitäten der NGOs auf, welche die staatlichen Behörden meist mit der Bekämpfung der Schlepperei rechtfertigen. ${ }^{31}$ Nachfolgend wird deshalb der Tatbestand der Schlepperei näher beleuchtet.

\section{Tatbestand der Schlepperei im Völkerrecht}

6 Auf internationaler Ebene wird der Schleppertatbestand hauptsächlich durch das sogenannte «Migrant Smuggling Protocol»32, einem Zusatzprotokoll des UN-Übereinkommens gegen die grenzüberschreitende organisierte Kriminalität33, geregelt. 34 Art. 3 lit. a des Protokolls definiert die «Schlepperei von Migranten» als «Herbeiführung der illegalen Einreise einer Person [...], mit dem Ziel, sich unmittelbar oder mittelbar einen finanziellen oder sonstigen materiellen Vorteil zu verschaffen». Der Tatbestand

3o $\overline{\text { Zwei Jahre und vier Monate nach der Verhaftung }}$ der fünf Mitarbeitenden der spanischen NGO «Proem-Aid», wurden sie schliesslich freigesprochen, dazu: The New York Times, Volunteers Who Rescued Migrants Are Cleared of Criminal Charges in Greece, 7. Mai 2018; zu den aktuellen Fällen der Strafandrohung für Personen, die sich an der Seenotrettung beteiligen, siehe auch: Siehe dazu: Lina Vosyliute, Is «Saving Lives at Sea» still a Priority for the EU?, 19. April 2018; vgl. Ghezelbash/Moreno-Lax/Klein/Opeskin (Fn. 28), S. 347.

31 Vgl. Ghezelbash/Moreno-Lax/Klein/Opeskin (Fn. 28), S. 347 ff.

32 Zusatzprotokoll vom 15. November 2000 gegen die Schleusung (Schweiz/Österreich: Schlepperei) von Migranten auf dem Land-, See- und Luftweg zum Übereinkommen der Vereinten Nationen gegen die grenzüberschreitende organisierte Kriminalität (SR 0.311.541).

33 Übereinkommen der Vereinten Nationen gegen die grenzüberschreitende organisierte Kriminalität vom 15. November 2000 (SR 0.311.54).

34 Anna T. Gallagher/Fiona David, The International Law of Migrant Smuggling, 2014, S. 44. 
der Schlepperei ist also nur dann erfüllt, wenn das Element der Bereicherung gegeben ist. 35 Ausweislich der Materialien zum Protokoll sollte dadurch die Unterstützung von Personen aus humanitären Gründen vom Tatbestand der Schlepperei ausgeschlossen werden. ${ }^{36}$

\section{Tatbestand der Schlepperei im EU-Recht}

7 Auf EU-Ebene wurde im Jahr 2002 das sogenannte «facilitators package» 37 erlassen, welches aus der Richtlinie 2002/90/EG38 zur Definition der Beihilfe zur unerlaubten Ein- und Durchreise und zum unerlaubten Aufenthalt, sowie einem Rahmenbeschluss des Rates 39 besteht.40 Gemäss Art. 1 Abs. 1 lit. a RL 2002/90/EG sollen die Mitgliedstaaten Personen sanktionieren, welche Drittstaatsangehörigen bei der irregulären Ein- oder Durchreise in den bzw. im EU-

35 Francis Webber, Humanitarianism: the unacceptable face of solidarity, Institute of Race Relations, 2017, S. 8; Ghezelbash/Moreno-Lax/Klein/ Opeskin (Fn. 28), S. 348; siehe dazu: Rachel Landry, The "humanitarian smuggling» of refugees, criminal offence or moral obligation?, working paper series no. 119, Refugee Study Centre Oxford, 2016, S. 8 ff.

36 Travaux Préparatoires of the UN Convention against Transnational Organized Crime and the Protocols thereto, S. 469; Ghezelbash/MorenoLax/Klein/Opeskin (Fn. 28), S. 348; Gallagher/ David (Fn. 34), S. 46; Landry (Fn. 35), S. 8.

37 Europäische Kommission, Towards a Comprehensive European Migration Policy: 20 years of EU Action, 4. März 2015; Landr (Fn. 35), S. 9.

38 Richtlinie 2002/90/EG vom 28. November 2002 zur Definition der Beihilfe zur unerlaubten Einund Durchreise und zum unerlaubten Aufenthalt, L 328/17.

39 Rahmenbeschluss 2002/946/JI des Rates vom 28. November 2002 betreffend die Verstärkung des strafrechtlichen Rahmens für die Bekämpfung der Beihilfe zur unerlaubten Ein- und Durchreise und zum unerlaubten Aufenthalt, L 328/1.

40 Gallagher/David (Fn. 34), S. 89; Webber (Fn. 35), S. 8.
Raum vorsätzlich helfen. ${ }^{41}$ Entgegen der völkerrechtlichen Bestimmung ist das Element der Bereicherung im EU-Recht somit keine explizite Bedingung, um unter dem Tatbestand sanktioniert bzw. kriminalisiert zu werden. $4^{2}$ Jedoch können die Mitgliedstaaten durch innerstaatliche Rechtsvorschriften jene Handlungen von der Sanktionierung ausschliessen, welche als Ziel die humanitäre Unterstützung der betroffenen Person verfolgen (Art. 1 Abs. 2 RL 2002/90/EG).43 Die Umsetzung dieser Schutzbestimmung für humanitäre Akteure in nationales Recht liegt im Ermessen der Mitgliedstaaten, was zur Folge hat, dass nur einige wenige Staaten eine solche in ihrem Recht auch tatsächlich verankert haben. 44 Unter anderem deshalb sind die Vorgaben des «facilitators package» - und dadurch auch die meisten nationalen Regelungen - nicht im Einklang mit den völkerrechtlichen Vorschriften. 45 Um dem Normzweck des «Migrant Smuggling Protocol» entgegenstehende Verurteilungen zu verhindern, ist zumindest eine Ausnahmeregelung von der Strafbarkeit für Personen, die aus humanitären Gründen bzw. ohne Bereicherungsabsicht handeln, erforderlich. ${ }^{6}$ Der europarechtliche Tat-

41 Ghezelbash/Moreno-Lax/Klein/Opeskin (Fn. 28), S. 347 f.; Webber (Fn. 35), S. 8.

42 Gallagher/David (Fn. 34), S. 89 und 91; Landry (Fn. 35), S. 10; Ghezelbash/Moreno-Lax/Klein/ Opeskin (Fn. 28), S. 348.

43 Gallagher/David (Fn. 34), S. 89; Landry (Fn. 35), S. 10.

44 Landry (Fn. 35), S. 10; siehe Europäisches Parlament, Briefing Bewertung der Umsetzung, Bekämpfung der Schleusung von Migranten in die EU, 16. April 2016, abrufbar, S. 8; vgl. Webber (Fn. 35), S. 19.

45 Mehr dazu: Gallagher/David (Fn. 34), S. 91 f.; Ghezelbash/Moreno-Lax/Klein/Opeskin (Fn. 28), S. $347 \mathrm{f}$.

46 Ghezelbash/Moreno-Lax/Klein/Opeskin (Fn. 28), S. 347 f.; vgl. Gallagher/David (Fn. 34), S. 91; vgl. Webber (Fn. 35), S. 8. 
bestand der Schlepperei birgt bei einer wortlautbasierten Auslegung der Rechtsnorm die Gefahr der Kriminalisierung der Seenotrettungsaktivitäten der NGOs und scheint deshalb als Droh- bzw. Druckmittel gegenüber den humanitären Akteuren verwendet zu werden. 47

\section{Regulierung der Aktivitäten der SAR NGOs}

\section{Der eigene Verhaltenskodex der SAR NGOs}

8 Bereits im Februar 2017 wurden von mehreren im Mittelmeer tätigen NGOs Anstrengungen unternommen, ihre SAROperationen $\mathrm{zu}$ koordinieren und das Misstrauen gegenüber ihren Aktivitäten zu vermindern, indem die Transparenz in den Tätigkeiten der NGOs erhöht wird.48 Der von zivilen NGOs vorgeschlagene freiwillige Verhaltenskodex für Such- und Rettungsaktionen im Mittelmeer 49 soll deshalb einen gemeinsamen ethischen Standard sowie das operationelle Vorgehen für effiziente, effektive und rechtmässige Such- und Rettungsoperationen definieren. ${ }^{\circ}$ Im Einzelnen betont der Verhaltenskodex die (Menschen-)Rechte, welche durch die Aktivitäten der SAR NGOs besser gesichert werden und und legt einen gewissen Minimalstandard betreffend das Einsatzverhalten sowie die Vorausplanung der Einsätze fest. ${ }^{11}$ Der zweite Teil widmet sich den leitenden humanitären

47 Landry (Fn. 35), S. 10 f.; Webber (Fn. 35), S. 8.

48 Vgl. Vorwort von David Hamond, CEO Human Rights at Sea, in: Voluntary Code of Conduct search and rescue operations undertaken by civil society Non-Government Organisations in the Mediterranean Sea, 1. Edition, Februar 2017, abrufbar unter, S. 2 (zit.: NGO-Verhaltenskodex).

49 NGO-Verhaltenskodex (Fn. 48).

50 Vgl. NGO-Verhaltenskodex (Fn. 48), S. 7 f.

51 Vgl. NGO-Verhaltenskodex (Fn. 48), S. $10 \mathrm{ff}$.
Grundsätzen der im Mittelmeer tätigen NGOs, namentlich die Menschlichkeit, die Unparteilichkeit, die Neutralität, die Unabhängigkeit und die Transparenz.52 Diese bilden einen wichtigen Bestandteil ihrer Identität als humanitäre NGOs und sollen ihnen eine gewisse Akzeptanz der lokalen Behörden sichern und dadurch ermöglichen, weitgehend unabhängig von der Politik zu agieren.53 Die Regulierung und Kontrolle der NGOs kann deshalb wenn dabei die Wahrung der humanitären Grundsätze in ihrer Tätigkeit gefährdet wird - im Konflikt zu ihrer eigentlichen Natur stehen, was am Beispiel des italienischen Verhaltenskodex' veranschaulicht werden kann.54

\section{Der italienische Verhaltenskodex für SAR NGOs}

9 Trotz der Anstrengungen der NGOs oder gerade als Reaktion auf diese - veröffentlichte die Europäische Kommission am 4. Juli 2017 den Vorschlag zum sogenannten «Aktionsplan zur Unterstützung Italiens, zur Verringerung des Migrationsdrucks und für mehr Solidarität»55. Dieser enthielt unter anderem die Aufforderung an Italien, einen Verhaltenskodex für SAR NGOs zu verfassen. Der Verhaltenskodex sollte in Beratung mit der Kommission und im Dialog mit den NGOs entstehen. $5^{6}$ Zwei Tage nach der Veröffentlichung des Vorschlags der

52 Vgl. NGO-Verhaltenskodex (Fn. 48), S. 8 ff.

53 Cusumano, humanitarian space (Fn. 29), S. 3 f.; vgl. NGO-Verhaltenskodex (Fn. 48), S. 8.

54 Vgl. Philip Alston/Ryan Goodman, International Human Rights, 2012, S. 1506.

55 Europäische Kommission, Action Plan on measures to support Italy, reduce pressure along the Central Mediterranean route and increase solidarity, 4. Juli 2017, (zit.: Europäische Kommission, Aktionsplan).

${ }^{6} 6$ Europäische Kommission, Pressemitteilung IP/17/1882, 4. Juli 2017. 
Kommission begrüssten die EUInnenminister im Rahmen einer informellen Tagung des Rates Justiz und Inneres in Tallinn die Idee eines italienischen Verhaltenskodex' für SAR NGOs, welcher allerdings in der Folgezeit eher als Initiative Italiens präsentiert wird.57

Der Verhaltenskodex 58 enthält 13 Punkte, zu deren Einhaltung sich die unterzeichnenden NGOs verpflichten sollten. $59 \mathrm{Ei}$ nige der Verpflichtungen stehen mit den genannten humanitären Grundsätzen der Menschlichkeit, der Unparteilichkeit, der Neutralität, der Unabhängigkeit und der Transparenz - in Konflikt. ${ }^{60}$ Die unterzeichnenden NGOs verpflichten sich zur Übermittlung angefragter Informationen an die italienischen Behörden zu Ermittlungszwecken, zur Kooperation mit den italienischen Behörden und der EU-Grenzschutzoperation Triton, sowie zur Duldung italienischer Polizisten an Bord von Rettungsschiffen, was insbesondere mit den humanitären Grundsätzen der Neutralität, der Unabhängigkeit und der Unparteilichkeit in Konflikt steht. 61 Auch das im Verhaltenskodex enthaltene Verbot der Einfahrt in liby-

57 Übersetzung aus dem Italienischen ins Englische: Code of Conduct for NGO undertaking activities in the migrants rescue operations at sea, 28. Juli 2017, S. 1 (zit.: Italienischer Verhaltenskodex); Cusumano, Straightjacketing (Fn. 29), S. 3.

58 Italienischer Verhaltenskodex (Fn. 57).

59 Die NGOs konnten jedoch Änderungen anfügen, wovon die unterzeichnenden NGOs auch Gebrauch gemacht haben; Cusumano, Straightjacketing (Fn. 29), S. 3 und 6; vgl. Italienischer Verhaltenskodex (Fn. 57), S. 1 ff.; vgl. Tagesanzeiger, Seenotretter wollen keine bewaffneten Polizisten an Bord, 2. August 2018.

60 Cusumano, Straightjacketing (Fn. 29), S. 5 f.

61 Vgl. Italienischer Verhaltenskodex (Fn. 57), S. 2 ff.; vgl. NGO-Verhaltenskodex (Fn. 48), S. 9; vgl. Cusumano, Straightjacketing (Fn. 29), S. 5 f.; vgl. Cusumano, humanitarian space (Fn. 28), S. 5 f.; Medecins sans frontiers, Q\&A: Why MSF didn't sign the Code of Conduct, 2. August 2017. sche Gewässer, sowie die Unterbindung der Telekommunikation und des Sendens von Lichtsignalen zur Erkennung des Standortes des Rettungsschiffes ist mit dem Grundsatz der Menschlichkeit nicht vereinbar. ${ }^{62}$ Die Verpflichtungen verletzen zudem teilweise auch völkerrechtliche Vorschriften. 63

11 Die Unterzeichnung durch die NGOs erfolgt formal «freiwillig», jedoch hält der Verhaltenskodex ausdrücklich fest, dass die fehlende Unterschrift zu «Massnahmen» gegenüber den betreffenden Schiffen führen kann. ${ }^{64}$ Trotz der Tatsache, dass die Androhung von Sanktionen welche die Verweigerung des Hafenzuganges beinhalten könnten 65 - unter den Vorbehalt der Vereinbarkeit mit internationalem Recht und dem Interesse der Rettung von Menschenleben gestellt ist, spricht die Sanktionsdrohung - schon aufgrund der geschilderten Unsicherheiten hinsichtlich der Rechtslage - gegen eine Freiwilligkeit. Die Androhung allfälliger Sanktionen gibt somit einen deutlichen Hinweis auf die Regulierungs- und

$62 \overline{\text { Der NGO-Verhaltenskodex hält unter dem Prin- }}$ zip der «Menschlichkeit» fest, dass die humanitäre Hilfe als Ziel die Rettung von Leben hat. Die Seenotrettung soll immer und überall erfolgen und nie eine politische oder parteiische Handlung darstellen, NGO-Verhaltenskodex (Fn. 48), S. 9; vgl. Italienischer Verhaltenskodex (Fn. 57), S. 2; vgl. Basaran (Fn. 17), S. 213; vgl. Cusumano, Straightjacketing (Fn. 28), S. 4.

63 Siehe dazu: Wissenschaftliche Dienste Deutscher Bundestag, Der Italienische Verhaltenskodex für private Seenotretter im Mittelmeer, Völker-, europa- und strafrechtliche Aspekte, 31. Juli 2017, S. 7 ff. (zit.: Wissenschaftliche Dienste Deutscher Bundestag, Der Italienische Verhaltenskodex).

64 Italienischer Verhaltenskodex (Fn. 57), S. 6.

65 Wissenschaftliche Dienste Deutscher Bundestag, Der Italienische Verhaltenskodex (Fn. 63), S. 5; ASGI, Position Paper on the Proposed «Code of Conduct for NGOs involved in Migrants' Rescue at Sea», 24. Juli 2017, S. 2; vgl. Cusumano, Straightjacketing (Fn. 29), S. 2 und 7. 
Kontrollabsichten Italiens gegenüber den SAR NGOs. 66

\section{Zur Vereinigungsfreiheit von SAR NGOs im Mittelmeer}

12 Das Zusammenspiel von Kriminalisierung und Regulierung(-sversuchen) der SAR NGOs erschwert deren Tätigkeit erheblich, insbesondere im Hinblick auf die Wahrung der humanitären Grundsätze ihrer Einsätze. ${ }^{67}$ Dies führt in Kombination mit der umstrittenen Zusammenarbeit zwischen der EU bzw. Italien und Libyen $^{68}$ und den daraus entstandenen Konflikten mit der «libyschen Küstenwache» $69 \mathrm{zu}$ einem starken Rückgang der Tätigkeiten der im Mittelmeerraum aktiven SAR NGOs.70 Tätigkeiten von NGOs fallen jedoch unter die v.a. in Art. 11 EMRK und Art. 12 GRC geschützte Ver-

$66 \overline{\text { ASGI (Fn. 65), S. 2; Hernan del Valle, Search and }}$ Rescue in the Mediterranean Sea, in: Oxford, Refugee Survey Quarerly, Vol. 35, 2016, S. 26; Webber (Fn. 35), S. 12; Basaran (Fn. 17), S. 213; vgl. Cusumano, Straightjacketing (Fn. 29), S. 6 f.

67 Vgl. Cusumano, humanitarian space (Fn. 29), S. 4.

68 Vgl. OHCHR, EU «trying to move border to Libya» using policy that breaches rights - UN experts, 17. August 2017; UN High Commissioner for Human Rights, Opinion by Zeid Ra'ad Al Hussein, Returned migrants are being robbed, raped and murdered in Libya, 8. September 2017, (zit.: Opinion by Zeid Ra'ad Al Hussein, UN High Commissioner for Human Rights); Nula Frei/Constantin Hruschka, Zur Umgehung des Refoulement-Verbots beim Kampf gegen «illegale Migration», in: Schweizerische Zeitschrift für Asylrecht und -praxis, 4/2017, S. 9 f.

69 «[...] a coast guard that has shot at NGO boats trying to rescue migrants at risk of drowning [...]», Opinion by Zeid Ra'ad Al Hussein, UN High Commissioner for Human Rights (Fn. 67); vgl. dazu: Wissenschaftliche Dienste Deutscher Bundestag, Die völkerrechtliche Pflicht zur Seenotrettung, 2017, S. 4 f; vgl. Frei/Hruschka(Fn. 68), S. $9 \mathrm{f}$.

70 Vgl. Frei/Hruschka (Fn. 68), S. 9 f. einigungsfreiheit. ${ }^{71}$ Die Effekte der versuchten Regulierung können demnach einen Eingriff in die Vereinigungsfreiheit der SAR NGOs darstellen, was nachfolgend geprüft werden soll.

13 Eine staatliche bzw. supranationale ${ }^{72}$ Massnahme weist Eingriffsqualität auf, wenn sie sich als abschreckend bzw. beeinträchtigend auf die Ausübung der garantierten Freiheit auswirkt, was gemäss dem Europäischen Gerichtshof für Menschenrechte (EGMR) selbst bei der Befürchtung einer weniger vorteilhaften Behandlung gegeben sein kann.73 Die Art bzw. die rechtliche Natur der Massnahme spielt dabei keine Rolle, sondern ausschliesslich deren Wirkung, weshalb auch der formal «freiwillige» Charakter einer Massnahme nichts an dessen Eingriffsqualität ändert.74 So kann gemäss der Rechtsprechung des EGMR bereits eine Aussage der öffentlichen Gewalt als Eingriff qualifiziert werden, wenn diese praktisch eine beeinträchtigende Wirkung auf die Ausübung der von der Vereinigungsfreiheit geschützten Aktivitäten hat.75

71 Jochen Frowein, Art. 11, in: Jochen Frowein/Wolfgang Peukert, die Europäische MenschenRechtsKonvention, EMRK-Kommentar, 3. Auflage, 2009, N 9; vgl. Arndt/Engels, Art. 11, in: Ulrich Karpenstein/Franz C. Mayer, EMRK, Konvention zum Schutze der Menschenrechte und Grundfreiheiten, Kommentar, 2. Auflage, 2015, S. 349 N 31.

72 Über Art. 51 i.V.m. Art. 52 Abs. 3 GRC bindet auch Organe und Institutionen der EU; vgl. Progin-Theuerkauf (Fn. 10), S. 73.

73 Birgit Daiber, Art. 11, in: Jens MeyerLadewig/Martin Nettesheim/Stefan von Raumer, EMRK, Handkommentar, 4. Auflage, 2017, S. 426 $\mathrm{N} 15$.

74 Vgl. Daiber (Fn. 73), S. 426 N 15.

75 Urteil des EGMR 11828/o8 vom 25. September 2012 (Trade Union of the Police in the Slovak Republik and others gegen Slowakei), N 60 und 61; Daiber (Fn. 73), S. 426 N 15. 
14 Der italienische Verhaltenskodex hat in Anwendung dieser Massstäbe Eingriffsqualität in Bezug auf die Vereinigungsfreiheit: Im Falle der Nichtunterzeichnung des Verhaltenskodex' müssen die NGOs Sanktionen befürchten, was sich in der Praxis auf die Möglichkeit zur Ausübung ihrer Tätigkeiten auswirkt. ${ }^{76} \mathrm{Im}$ Falle der Unterzeichnung des vorgesehenen Verhaltenskodex', müssen sie sich an die vereinbarten - und teilweise mit ihren humanitären Prinzipien in Konflikt stehenden - Regelungen halten, welche sich ebenfalls einschränkend auf ihre Tätigkeiten auswirken.77

15 Eingriffe in die Vereinigungsfreiheit können gerechtfertigt werden und stellen - sofern der Wesensgehalt unangetastet bleibt - nicht per se eine Verletzung von Art. 11 EMRK dar. ${ }^{78}$ Dafür müssen die in Art. 11 Abs. 2 EMRK festgelegten Bedingungen kumulativ erfüllt sein.79

Nach Art. 11 Abs. 2 EMRK muss die staatliche Massnahme zunächst gesetzlich vorgesehen sein. Da die rechtliche Grundlage für den Erlass des italienischen Verhaltenskodex' nicht ersichtlich ist, fehlt es bereits an diesem Erfordernis. ${ }^{80}$

Zudem muss der Eingriff ein in Art. 11 Abs. 2 EMRK genanntes legitimes Ziel verfolgen und die Massnahme in einer demokratischen Gesellschaft notwendig - d.h. verhältnismässig - sein. ${ }^{81}$ Als legitime Ziele kommen der Schutz der natio-

\footnotetext{
76 Vgl. Cusumano, Straightjacketing (Fn. 29), S. 3.

77 Vgl. Cusumano, humanitarian space (Fn. 29), S. 2.

78 Daiber (Fn. 73), S. 429 f. N 22.

79 Daiber (Fn. 73), S. 429 f. N 22.

80 Wissenschaftliche Dienste Deutscher Bundestag, Der Italienische Verhaltenskodex (Fn. 63), S. 13.

81 Grabenwarter/Pabel, Europäsche Menschenrechtskonvention, 6. Auflage, 2016, N 92.
}

nalen und öffentlichen Sicherheit, die Aufrechterhaltung der Ordnung, die Verhütung von Straftaten, der Schutz der Gesundheit oder der Moral, oder der Schutz der Rechte und Freiheiten anderer in Frage. ${ }^{82}$ Selbst wenn der staatliche Eingriff unter eines der legitimen Ziele subsumiert werden könnte, wie beispielsweise im Rahmen des «Kampfs gegen Schleuser» 83 unter die Verhütung von Straftaten, ist er unverhältnismässig. Der Verhaltenskodex wäre im «Kampf gegen Schleuser» weder geeignet noch notwendig, da die Tätigkeiten der SAR NGOs keinerlei Verbindung zu Schleppertätigkeiten aufweisen und zur Verhütung einer solchen Verbindung beispielsweise auch ein freiwilliger Verhaltenskodex ausreichen würde, der die Verpflichtung enthält, nicht mit Schleppern zusammenzuarbeiten. ${ }^{84}$ Zudem stellt dieser subtile Regulierungsversuch die NGOs vor die Wahl, in partieller Verletzung ihrer humanitären Prinzipien zu kooperieren oder eine Kriminalisierung ihrer Tätigkeiten zu riskieren. 85 Die Einhaltung der humanitären Prinzipien fällt jedoch für die NGOs unter den Wesensgehalt ihres Daseins. ${ }^{86}$ Obwohl die Vereinigungen im Mittelmeer nach wie vor tätig sein können, ähneln die Wirkungen einer solchen Regulierung einem Verbot

$82 \overline{\text { Grabenwarter/Pabel (Fn. 81), N 92; }}$ Daiber (Fn. 73), S. 429 f. N 22.

83 Aussage Jean-Claude Junker, Europäische Kommission, Aktionsplan (Fn. 55); Vgl. Violeta MorenoLax, The EU Humanitarian Border and the Securitization of Human Rights: The «Rescue-ThroughInterdiction/Rescue-Without-Protection» Paradigm, in: Journal of Common Market Studies, 56, 2018, S. 128 und 133 (zit.: Moreno-Lax, RescueThrough-Interdiction/Rescue-Without-Protection).

84 Vgl. Cusumano, Straightjacketing (Fn. 29), S. 2 f. und $7 \mathrm{f}$.

85 Vgl. Cusumano, humanitarian space (Fn. 29), S. 6; vgl. Cusumano, Straightjacketing (Fn. 29), S. 2.

86 Vgl. Alston/Goodman (Fn. 54), S. 1506. 
der Tätigkeit als NGO. ${ }^{87}$ Sie riskieren durch die Kooperation mit den staatlichen Behörden ihre Identität als unabhängige, humanitäre Organisation $\mathrm{zu}$ verlieren. ${ }^{88}$ Gerade aufgrund dieser Unabhängigkeit kommt den NGOs im politischen Diskurs jedoch eine wichtige Rolle zu, da sie oftmals eine Gegenstimme zu ausschliesslich staatlichen Interessen bilden. 89

Wird die Tätigkeit der SAR NGOs an Bedingungen geknüpft, die den humanitären Prinzipien entgegenstehen, kommt dies einem Verbot oder einer Nichtzulassung der Vereinigung gleich, an welche der Gerichtshof hohe Anforderungen stellt.90 Namentlich müssen konkrete Anhaltspunkte bestehen, dass die in Art. 11 Abs. 2 EMRK genannten Rechtsgüter durch die Ziele oder Aktivitäten der Vereinigung gefährdet werden, was im Falle der Seenotrettung durch zivilen Organisationen im Mittelmeer nicht der Fall ist, da keinerlei Verbindungen $\mathrm{zu}$ Schleppertätigkeiten bestehen. ${ }^{91}$

Im Rahmen der Verhältnismässigkeitsprüfung muss zudem eine gesamtheitliche Abwägung zwischen betroffenen Rechten und Rechtsgütern stattfinden.92 Bei der Regulierung der Aktivitäten der SAR NGOs fällt dabei insbesondere das

87 Vgl. Daiber (Fn. 73), S. 428 f. N 19.

88 Siehe Jugend Rettet: «That is antithetical to the humanitarian principles of neutrality that we adhere to [...]» in: The Guardian, Aid groups snub Italian code of conduct on Mediterranean rescues, 31. Juli 2017.

89 Dies ist wichtig, da der EGMR bei der Beurteilung der Verhältnismässigkeit des Eingriffs die Bedeutung der Vereinigung für das Funktionieren der Demokratie berücksichtigt, Arndt/Engels (Fn. 71), S. 350 N 35.

90 Vgl. Arndt/Engels (Fn. 71), S. 351 N 39.

91 Arndt/Engels (Fn. 71), S. 351 N 39.

92 Arndt/Engels (Fn. 71), S. 350 N 35. fundamentale Menschenrecht auf Leben bzw. der Schutz des Lebens der Personen in Seenot ins Gewicht. 93 Problematisch ist dabei, dass die Rettung von Menschenleben auch als (rechtlich und faktisch nicht überzeugendes)94 Argument für Regulierungs- und sogar Restriktionsversuche der Seenotrettungsaktionen benutzt wird, indem argumentiert wird, dass hierdurch ein "pull factor» wegfällt und sich somit weniger Leute auf den gefährlichen Weg über das Mittelmeer machen.95 Tatsächlich ist so ein Effekt jedoch nicht nachweisbar. 96 Die einschränkenden Massnahmen führen jedoch dazu, dass die NGOs in der Praxis daran gehindert werden, Menschenleben im Mittelmeer zu retten. 97 Unter anderem dadurch steigt die Anzahl der Todesfällen vor der libyschen Küste spürbar an. ${ }^{8}$ Der Staat - in diesem Fall Italien verletzt somit seine positive Schutzplicht, die ihm aus dem Recht auf Leben (Art. 2 EMRK) erwächst.99

2o Abschliessend kann festgestellt werden, dass auch die Europäische Kommission im Rahmen der Regulierung von NGOs

93 Siehe dazu: Komp (Fn. 71), S. 236 ff.

94 Ausführlich und mit passendem Beispiel veranschaulicht in Frei/Hruschka (Fn. 68), S. 9; vgl. MEDMIG, Unpacking a rapidly changing scenario: migration flow, routes and trajectories across the Mediteranean, 1. März 2016, S. 10.

95 Frei/Hruschka (Fn. 68), S. 9; vgl. Moreno-Lax, Rescue-Through-Interdiction/Rescue-WithoutProtection (Fn. 83), S. 133.

96 MEDMIG zeigt auf, dass SAR Operationen keinen «pull-effect» haben, vgl. dazu: MEDMIG, (Fn. 94), S. 10.

97 Vgl. Cusumano, Straightjacketing (Fn. 29), S. 7 f.

98 Vgl. UNHCR, appalled by the loss of life at sea off the coast of Libya, 30. Juni 2018.

99 Isabel Schübel-Pfister, Art. 2, in: Ulrich Karpenstein/Franz C. Mayer, EMRK, Konvention zum Schutze der Menschenrechte und Grundfreiheiten, Kommentar, 2015, S. 74 ff. N 30 ff.; ProginTheuerkauf (Fn. 10), S. 72; vgl. Cusumano, Straightjacketing (Fn. 29), S. 4. 
die erhebliche Bedeutung der Zivilgesellschaft als «Gerüst unserer demokratischen Gesellschaft»100 anerkennt. ${ }^{101} \mathrm{Im}$ Juli 2017 leitete sie deshalb ein Vertragsverletzungsverfahren gegen Ungarn wegen dessen neu verabschiedetem Gesetz ein, welches nichtstaatliche Organisationen, die Gelder aus dem Ausland erhalten, verpflichtet sich zu kennzeichnen. ${ }^{102}$ Dabei betont die Kommission, dass das Gesetz insbesondere auch gegen das in der Grundrechtscharta verankerte Recht auf Vereinigungsfreiheit verstosse, da es die NGOs an der Geldeinwerbung und der Ausübung ihrer Aufgaben hindere. ${ }^{103}$ Dass Italien im selben Zeitraum zu einer (einschränkenden) Regulierung der Tätigkeiten der SAR NGOs im Mittelmeer aufgefordert wurde, ist mit diesen Vorbringen nicht kohärent.

\section{Schlussfolgerungen}

21 Der vorliegende Beitrag hat gezeigt, dass die Situation von SAR NGOs im Mittelmeer rechtlich und tatsächlich immer komplexer wird. Trotz und zum Teil wegen ihrer wichtigen Rolle in der Rettung von Menschenleben und einer bestehenden völker- und menschenrechtlichen Pflicht zur Seenotrettung werden die Aktivitäten der NGOs sowohl von der

100 Aussage von Frans Timmermans, Erster Vizepräsident der Kommission. Europäische Kommission, Pressemitteilung IP/17/1982 vom 13. Juli 2017.

101 Vgl. Pressemitteilung IP/17/1982 (Fn. 100).

${ }^{102}$ Am Beispiel von Ungarn kann veranschaulicht werden, wie solche Entwicklungen zu einer umfassenden Kriminalisierung der NGOs, die Geflüchtete unterstützen, führen können: Im Juni 2018 wurde das sogenannte «Stop-Soros»Gesetzespacket verabschiedet, welches Hilfeleistungen für geflüchtete Menschen in Ungarn weitgehend kriminalisiert; vgl. NZZ, Ungarn kriminalisiert Flüchtlingshilfe, 20. Juni 2018.

103 Vgl. Pressemitteilung IP/17/1982 (Fn. 100).
Kommission selbst als auch von den Mitgliedstaaten praktisch erheblich erschwert, wenn nicht sogar verunmöglicht. Diese und weitere Massnahmen der EU führen dazu, dass der Weg von und durch Libyen über die zentrale Mittelmeerroute nach Europa immer gefährlicher wird und fundamentale Rechte der geflüchteten Personen verletzt werden.104 Sichere Zugangswege fehlen, weshalb die Flucht übers Mittelmeer oftmals die einzige noch verbleibende Möglichkeit ist, nach Europa zu gelangen und einen Antrag auf internationalen Schutz zu stellen. Der Zugang zu Schutz in Europa wird dadurch erheblich erschwert bzw. vielen Menschen faktisch verwehrt.

22 Das Zusammenspiel von Kriminalisierung und Regulierung der Aktivitäten der zivilgesellschaftlichen Seenotrettungsorganisationen im Mittelmeer führt dazu, dass deren Aktionsradius und Handlungsfähigkeit erheblich verringert wurden. Den SAR NGOs wird es nahezu verunmöglicht, ihren Tätigkeiten unter Wahrung der humanitären Prinzipien nachzugehen. Diese Situation stellt einen Eingriff in die Vereinigungsfreiheit dar, auf welche sich die SAR NGOs berufen können. Die Prüfung des italienischen Verhaltenskodex' hat ergeben, dass staatliche und supranationale Regulierungsversuche, die faktische Abhängigkeiten schaffen und die SAR NGOs dadurch in eine Zwangslage versetzen, eine Verletzung der Vereinigungsfreiheit gemäss Art. 11 EMRK darstellen, da sich die NGOs zunehmend von ihrer eigentlichen Natur entfernen müssen, um im Sinne des Kodex' im Mittelmeer aktiv bleiben zu können.

$104 \overline{\text { Vgl. Opinion by Zeid Ra'ad Al Hussein, UN High }}$ Commissioner for Human Rights (Fn. 67). 
23 Wie den Vorbringen der Kommission bezüglich der Regulierung der NGOs im oben genannten Vertragsverletzungsverfahren gegen Ungarn zu entnehmen ist, spielen die zivilgesellschaftlichen Akteure und deren Recht auf Vereinigungsfreiheit eine wichtige Rolle im europäischen Wertesystem, das sich in der Grundrechtecharta und der EMRK spiegelt. Im Diskurs über die Regulierung der SAR NGOs auf der zentralen Mittelmeerroute finden diese Argumente jedoch keine relevante Berücksichtigung. In Anbetracht des politischen Kontextes der Massnahmen wird hier wohl mit zweierlei Mass gemessen: Im Rahmen des von der EU Kommission formulierten Ziels der «Verringerung der Migrationsströme»105 scheint die EU keine Mittel zu scheuen und bereit zu sein, selbst den Schutz fundamentaler Menschenrechte bzw. die Rettung von Menschenleben in den Hintergrund treten zu lassen. 